\title{
How Smokers Change Their World and How the World Responds: \\ Testing the Oscillatory Nature of Micro-psychokinetic Observer Effects on Addiction-related Stimuli
}

\author{
Moritz C. Dechamps \\ Markus A. Maier \\ Department of Psychology \\ Ludwig-Maximilians-University, Munich, Germany
}

Moritz C. Dechamps, M. Sc. of Psychology

Dr. Markus A. Maier, Professor of Psychology

Department of Psychology,

Ludwig-Maximilians-University, Munich, Germany

Correspondence concerning this article should be addressed to

Moritz C. Dechamps M. Sc. or Prof. Dr. Markus A. Maier, Department of Psychology,

Leopoldstr. 13, Ludwig-Maximilians-University

80802 München

Email: moritz.dechamps@psy.lmu.de

Competing Interests: No competing interests were disclosed. 


\section{Abstract}

According to standard quantum theory, the occurrence of a specific outcome during a quantum measurement is completely random (see Bell 1964). However, some authors refer to revised versions of quantum mechanics (e.g., Penrose \& Hameroff 2011, Mensky 2013, Stapp 2017), and propose that the human mind can actually influence the probability of such outcomes. Empirical support for this idea has been provided by micro-psychokinesis (microPK) research (see Bösch, Steinkamp, \& Boller 2006). However, attempts to replicate these findings have often failed (e.g. Jahn et al. 2000). In an attempt to explain these failures, von Lucadou, Römer, and Walach (2007) established a theoretical model for predicting unsystematic variations of such an influencing effect across replications, resulting in a decline of the effect in micro-PK data over time. Maier, Dechamps, and Pflitsch (2018) slightly expanded this theory by proposing that the temporal variation of such an effect follows a systematic pattern, which can be tested and used for prediction making. In this research we generated such a prediction using data from two previous studies that initially demonstrated a strong micro-PK followed by a subsequent decline in the effect over the course of 297 participants (Maier \& Dechamps 2018), we then put it to test with an additionally preregistered set of recollected data from 203 subjects. We compared these results with 10,000 simulated data sets (each set with an $N=203$ ) each comprising random data. Three tests were applied to the experimental data: an area under the curve analysis, a local maximum fit test, and an endpoint fit test. These tests revealed no significant fit of the real data regarding the predicted data pattern. Further analyses explored additional techniques, including an analysis of the highest reached Bayes Factor $(B F)$ over the course of the experiments, the overall orientation of the $B F$ curve, and its transformation into oscillatory components via a Fourier analysis. All these methods allowed for statistically significant differentiations between experimental data on the one side, and the control group and 
simulation data on the other. We conclude that the analyses of the temporal development of an effect along these lines constitute a fruitful approach toward testing non-random and volatile time trends within micro-PK data.

Keywords: micro-psychokinesis, observer effect, quantum measurement, mind-matter 


\section{Introduction}

The relationship between the mental and the physical worlds, between mind and matter, remains a fascinating enigma to this date. Recently, advanced theories have tried to shed light on the mutual interactions of these worlds by incorporating ideas pertaining to an inherent connection of quantum theory and consciousness (see Atmanspacher 2004). A quantum state is characterized by peculiar properties, such as the superposition of multiple states or the possibility of entanglement. The transition from a quantum to a classical state is associated with the measurement of the quantum system. Comparatively, orthodox quantum mechanics mostly denies that mental aspects play a crucial role in the measurement process (see Greenstein \& Zajonc 2006); some researchers argue that consciousness, or other mental concepts, comprise a much more relevant part in this transition (e.g., Penrose \& Hameroff 2011; Mensky 2013; Stapp 2017; see also von Neumann 1932; Wigner 1963).

According to some of these models, the quantum state can be interpreted as a pre-real possibility space, which interacts with both classical physical states and their corresponding representations within the conscious mental world (Filk \& Römer 2011; Atmanspacher \& Filk 2012; Stapp 2015). Quantum measurement, the moment when one of many superposed possibilities becomes real according to classical physics, is equally considered as a transference of knowledge from unknown into consciously known knowledge. If such a measurement is characterized by the conscious processing of a classical outcome, quantumspecific effects must therefore operate within a pre-conscious realm (Penrose \& Hameroff 2011; see also Penrose 1989, 1994; Hameroff \& Penrose 1996; Hameroff 2012). Accepting this possibility, Jung and Pauli (see Atmanspacher 2012) even argued that conscious observation during quantum measurements does not causally influence the physical transition from quantum to classical states; rather, conscious observation of a measurement result cooccurs with the manifestation of a classical state in the physical world. Although standard 
orthodox quantum theory predicts the occurrence of specific measurement outcomes with a probability function that is ontic in nature, the aforementioned alternative approaches allow for mental influences on these likelihoods (e.g. Stapp 2015). Maier and Dechamps (2018) identified the transition from the unconscious to a conscious state of mind as a candidate for biasing quantum probabilities. Accordingly, unconscious motivational states, which translate their drives via emotions (e.g., fear or hope) into conscious realizations, have been proposed as a central reality-shaping factor. For instance, if an individual has an unconsciously grounded motive to smoke, their corresponding desire is emotionally laden with the fear of not having enough nicotine in their body. This deficit-oriented fear translates into an expectation of never receiving enough of the desired substance. When such an addicted person interacts with a quantum system that is connected with two equally likely realitiesone that corresponds to their motive, and the other being neutral regarding their motive - their desire will influence the measurement of that quantum system. Accordingly, this will lead to an increased likelihood of experiences corresponding to the implicit belief; a consequence that standard quantum probability would not allow. In other words, under such circumstances the unconscious mental constitution of an observer would create classical realities that include their conscious observations that accord with the individual's observer's inner emotional desires. This would represent a truly self-fulfilling prophecy.

This idea is congruous with results yielded by micro-psychokinetic (micro-PK) research. Micro-PK can be described as mental influences on inanimate and probabilistic systems that produce effects that are only detectable through statistical means (Varvoglis \& Bancel 2015). Typically, this involves studies measuring the influence of observers on random target systems, such as random number generators. Several meta-analyses have aggregated data from hundreds of micro-PK studies and found substantial overall effects resulting from observer influences (Radin \& Nelson 1989; Bösch, Steinkamp, \& Boller, 2006). The high heterogeneity of the effect sizes raises concerns for some critics, however, 
Varvoglis and Bancel (2015) conclude that despite some publication bias quite possibly being present, an unrealistically large file drawer would be needed to annul the results (see also Radin et al., 2006).

Nevertheless, micro-PK research has to grapple with recurrent difficulties, arguably the most important of which is the lack of successful direct replications (e.g. the replication of the PEAR landmark study; Jahn et al. 2000). This evasive nature of psi effects has not gone unnoticed within the psi research community (Kennedy 2003), and has furthermore confronted us with challenges during an attempt to replicate a promising first study on microPK (Maier \& Dechamps 2018).

In this set of two consecutive studies, we originally aimed to investigate a possible mind-matter interaction by experimentally linking the outcome of a random event to a psychologically meaningful experience. Studies focusing on a relaxed mental state of effortless intention usually generate better results than those focusing on an intentional and deliberate influence on randomness (e.g. Braud \& Braud 1979; Debes \& Morris 1982). Accordingly, we designed our independent variable using a primarily unconsciously driven state: the desire for cigarettes within regular smokers. Through these means we ensured to integrate the subject's implicit mental state into the design.

In the first study, we compared the effects of participants with a pronounced driveregular smokers - on the output of true random number generators (tRNGs) with the effects of non-smoking, and therefore unmotivated, participants. Depending on the tRNG's outcome, pictures pertaining to the drive and need (e.g. people smoking) or pictures not pertaining to this need (e.g. a chair) were displayed to the passively observing participants. We then tested whether the distinctive mindset of regular smokers led to a bias in the quantum measurement process and, subsequently, to a non-random distribution of addiction-relevant stimuli. 
In study 1, 122 smokers and 132 non-smokers were presented with 400 trials. We hypothesized that the specific mental attitude toward cigarette-related content within smokers would lead to a deviation in the number of smoking pictures from that of random chance; accordingly, no deviation from chance was expected concerning the non-smokers.

Data were analyzed using a Bayesian approach (see Wagenmakers et al. 2011), which updates the support and evidence for either hypotheses with each new data point by pitting their likelihoods against one other. This subsequently allows for sequential testing, which involves adding and continuously analyzing data until a pre-specified stopping criterion has been met. We decided upon a Bayes Factor $(B F)$ of 10 as a stopping rule, which corresponds to strong evidence toward a given hypothesis. Concerning a prior probability, we decided to use a Cauchy distribution centered on zero with an $\mathrm{r}$ of 0.5 , i.e. $\delta \sim$ Cauchy $(0, .5)$, as proposed by Bem, Utts, and Johnson (2011). At the beginning of this research there was no clear prediction regarding direction of the effect, and so a two-sided approach was chosen.

The final Bayesian t-test analysis with 122 smokers yielded a $B F$ of 66.06 towards $\mathrm{H}_{1}$; this means that it was 66 times more likely to obtain the data when the alternative hypothesis was true than when the null-hypothesis was correct. Smoking participants saw an average of 49.18\% addiction-related stimuli ('cigarette pictures'), a percentage substantially below the expected chance value of $50 \%$, thereby representing a very strong effect. Concerning the nonsmoking subsample of 132 participants, the Bayesian t-Test yielded a $B F$ of 6.13 towards $\mathrm{H}_{0}$; this indicates moderate evidence for a null effect (see Table 1). These remarkable results point to a need-specific micro-PK effect within those participants who experienced a

subconsciously active but deficit-oriented desire toward the relevant stimuli. The results are in line with the reasoning of the emotional transgression model, as outlined above (see also Maier, Dechamps, \& Pflitsch 2018). This model emphasizes the importance of implicit beliefs - in this case an unconscious experience of a lack of nicotine - when influencing the 
actualization of a corresponding outcome as part of a micro-PK experiment. This deficit translates into craving and addictive actions on one side, and into a subconsciously active mental pattern centering on 'not having enough' (of the addictive substance) on the other. Consequently, this unconscious fear leads to a self-fulfilling prophecy: the lack experienced by the smoker 'establishes' a confirming reality that misses smoking-related content. Accordingly, consciously experienced classical reality corresponds to the unconscious inner beliefs of the observer.

After these initially promising results, we decided to perform an exact replication of the experiment to further substantiate the observed effect. The pre-registered replication ${ }^{1}$ used the same procedural details as those of the first study. Surprisingly, our expectations were not met, and study 2 did not replicate the findings of study 1 . For smokers, study 2 revealed strong evidence towards the null hypothesis $\left(B F_{01}=11.07\right)$; comparatively, for non-smokers study 2 revealed moderate evidence in the same direction, replicating the findings of study 1 (see Table 1).

We faced a dilemma at this point, as the data yielded by study 2 suggested there was no replicable effect of the smoker subsample on the number of cigarette pictures. Still, the effect development of the smoker and non-smoker subsamples seemed to differ immensely over the course of both experiments. This was particularly obvious when concatenating the data of both studies, as is allowed with Bayesian sequential testing. The two-sided one-sample t-tests showed a remarkable development within the smoker subsample. The $B F$ begins to rise after about 80 participants, quickly surpassing the threshold of (very) strong evidence, reaching a climax of $B F_{10}=421.2$ at participant number 134 , shortly after commencing the second study. Soon a dramatic decline began to appear, countering the effect and eventually

\footnotetext{
${ }^{1}$ Preregistration available at https://osf.io/z7c4y/
} 
leading to an overall $B F$ lower than 1, indicating evidence toward the null hypothesis. This extraordinary change in effect across times was found only within the smoker subsample. Contrarily, the non-smoker subsample showed a steady and consistent decline towards $\mathrm{H}_{0}$ from the beginning (see Figure 1).

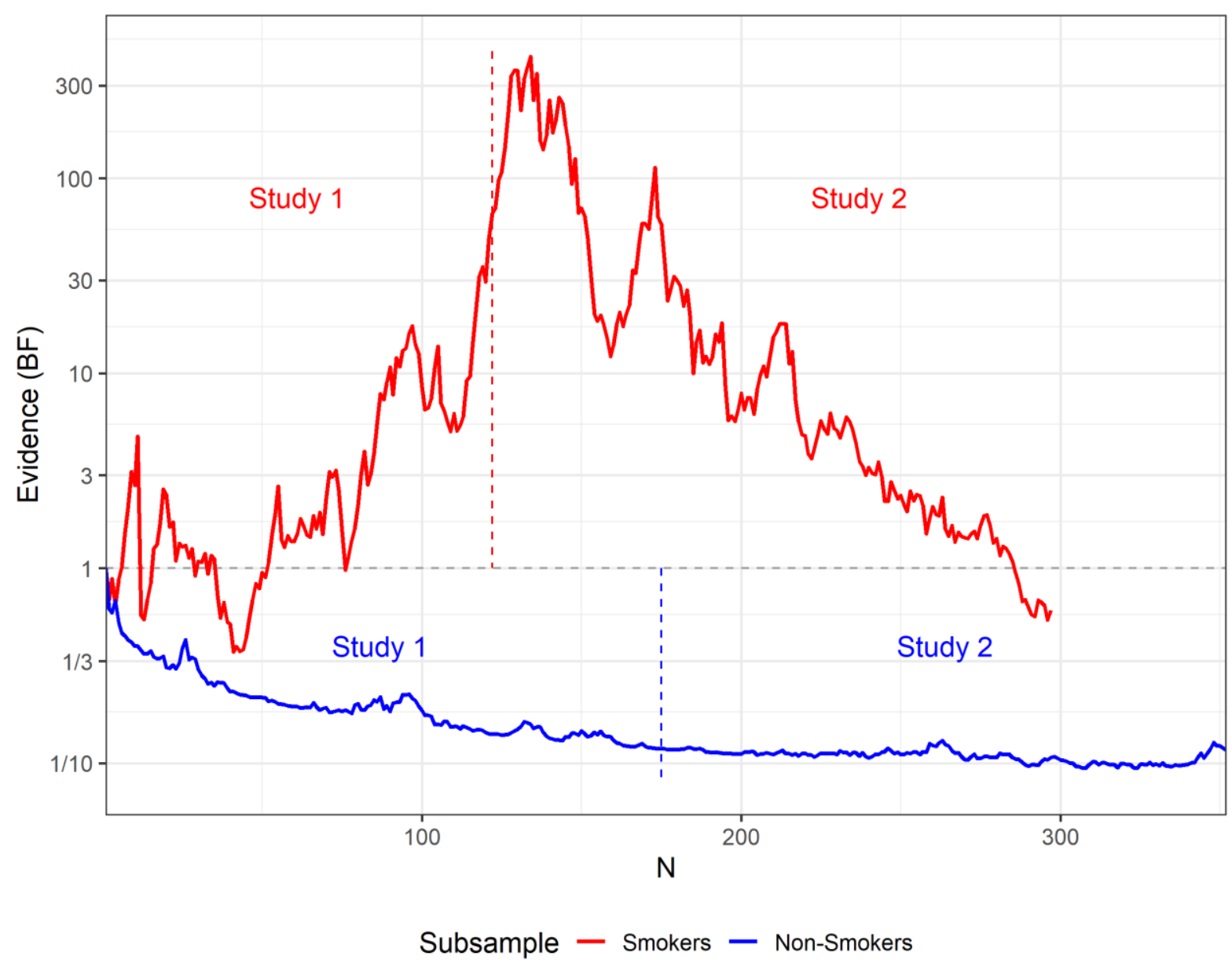

Given the apparent differences of the temporal changes in the sequential $B F$ s of the two subsamples, we speculated as to the presence of a systematic time-dependent micro-PK effect, despite the lack of replication. As mentioned earlier, decline effects are somewhat prominent, especially in psi research (e.g. Bem, Palmer, \& Broughton 2001; Radin 2006). Accordingly, it seems as if for these - and potentially other effects - different methodological approaches might be used other than the replication of original results. As the temporal development of the effect is so clearly identifiable among the data, we decided to focus on this temporal 
change. Of particular interest was the onset and development of the effect decline and whether a systematic pattern could be described here (see also Maier, Dechamps, \& Pflitsch 2018).

This decline of initially strong effects — we refer to this pattern as the "Nemesis effect" (in reference to the ancient Greek goddess of retributive justice)-seems to pervade in many psi studies, within both our lab and others. Von Lucadou, Römer and Walach (2007) addressed this phenomenon theoretically and to our knowledge for the first time in psi history, providing an elaborated explanation in their Model of Pragmatic Information (see also von Lucadou 2006, 2015). Pragmatic information is meaningful for a closed system and provides an opportunity for entropy-reducing change (see Weizäcker 1974). The authors considered this kind of pragmatic information as a prerequisite for the emergence of non-local correlations within closed psychophysical systems, which underlie most psi effects (von Lucadou 1995; Walach, von Lucadou, \& Römer 2014). Psi effects can be understood as entanglement correlations that are mediated not through causal signals, but rather as a consequence of a specific configuration of a system's mental and material components (von Lucadou, Römer, \& Walach 2007). Any artificial setups allowing the use of such pragmatic information within entanglement correlations for signal transmission-something that would be feasible if psi effects were robust and replicable — would lead to a reduction of pragmatic information and, therefore, to an unsystematic disappearance of psi effects in later replication attempts. This would satisfy the important Non-Transmission-Axiom (NT-Axiom) of quantum mechanics (Atmanspacher, Römer, \& Walach 2002; von Lucadou et al. 2007). The NT-Axiom is supposed to prevent physical paradoxes such as faster-than-light signal transfer (see also Einstein, Podolsky \& Rosen 1935), and ensure that natural order is abided (Walach, von Lucadou, \& Römer 2014). Von Lucadou, Römer and Walach (2007) condensed those factors responsible for the decline in the following formula:

$$
I=R * A=B * E
$$


Here, the amount of pragmatic Information (I) increases with higher proportions of Novelty (E) and Autonomy (A), and decreases with higher proportions of Confirmation (B), and Reliability (R). Replication studies involve low levels of novelty and autonomy as they often involve the specification of a clear prediction and are, therefore, unlikely to contain a sizeable amount of pragmatic information; i.e. they are without unambiguous results (Etzold 2004; Walach, von Lucadou, and Römer 2014). In sum, the Model of Pragmatic Information (MPI) predicts that exact replication attempts of psi effects based on non-locally correlated systems need to decline in the original dependent variable and possibly re-appear unpredictably in other variables of the system (so called 'displacement'). This would also be true for micro-PK effects and may provide an explanation for the elusiveness of such phenomena (von Lucadou, Römer, \& Walach 2007).

Maier, Dechamps and Pflitsch (2018; see also Maier \& Dechamps 2018) also refer to the MPI to explain their results, though they revised its predictions regarding the decline of psi effects. Instead of adopting the assumption regarding the unsystematic disappearance of the effect per the original MPI, these researchers proposed a systematic temporal change of micro-PK effects (and psi effects in general). This revision is based on the idea that evidence for micro-PK constitutes a severe violation of the Second Law of Thermodynamics because a chaotic system, such as a random quantum event becomes more orderly under micro-PK without the investment of energy. Such a system therefore decreases in entropy over time. Consequently, with increasing evidence for micro-PK, the onset of an entropic counter-effect is proposed. We assumed that this entropic counter-effect manifests as soon as the information resulting from mentally-induced deviations from quantum randomness reaches a certain threshold of experimental evidence. Once this original effect-and the evidence for such an effect—has weakened, the counter-effect also loses power, allowing the micro-PK effect to reappear again. This interplay between effect and counter-effect should lead to a distinctive pattern when analyzing the effect's temporal development. 
In a recent publication reporting the results of two micro-PK studies using smokers and non-smokers - and the time course of these effects across the studies (Maier \& Dechamps 2018) — we propose a way to study a systematic variation of the effects by analyzing their temporal change. Within the aforementioned publication we argued that psi effects will display a systematic interplay of effect and counter-effect in the form of a dampened harmonic oscillation. We then applied this idea to the smokers' data from studies 1 and 2. To do this, we plotted the effect as a cumulative z-score and used a curve-fitting algorithm, using the software Wolfram Mathematica Version 11.1 (https://www.wolfram.com/mathematica), estimating a dampened harmonic oscillation as an approximation of the raw data. Furthermore, an extrapolation of this function was then used to predict the future time course of a third set of data with that of prospectively collected micro-PK data (the study reported herein). For this purpose, the extrapolated continuation trend of our calculated curve should constitute a rough estimate for the development of the effect within this future data set (see Figure 2). 


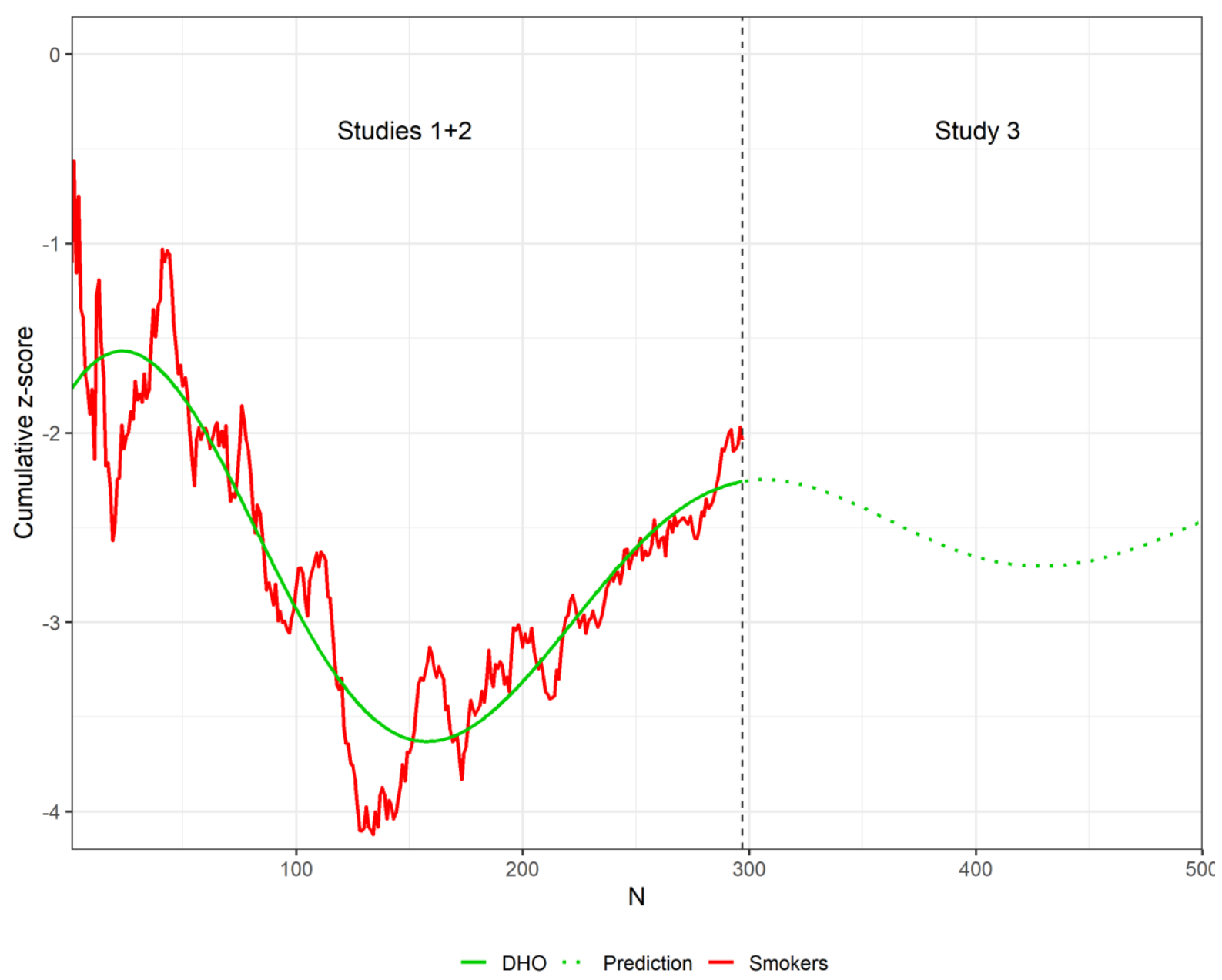

From this curve extrapolation a prediction for the next 200 participants can be derived: After a temporary local minimum (technically a local maximum, as the effect is negative), the effect will reappear, though with a lower effect size and then decline once more to a value similar or slightly more negative than the initial end-point value. The local maximum should appear around subject number 410-450, and should lie between a z-score of -2.5 and -3 . We decided to look at several test statistics to test for the quality of our prediction among the experimental data compared to 10,000 simulated data sets:

1) The area between the experimental data curve and the prediction.

2) The Euclidian distance of the local maximum compared to that of the prediction.

3) The Euclidian distance of the end point compared to that of the end point of the prediction. 


\section{Methods}

This study was pre-registered at the Open Science Framework (OSF)

(https://osf.io/ac839). It was conducted in accordance with the ethical requirements of the American Psychological Association (APA). All materials and procedural decisions were identical to those of studies 1 and 2 (Maier \& Dechamps 2018).

\section{Consent}

All participation in this study was voluntary, and written consent was obtained from all participants prior to their involvement. Interested participants were given an individual explanation about the purpose of this study once they had completed their tasks. The procedure used in this study was approved by the ethical board of the Department of Psychology.

\section{Participants}

Participants were added until a total of 500 smokers had been reached, including all participants from studies 1 and 2. This resulted in a total of 236 tested participants for this study; of these participants, 203 were smokers and were included in this study accordingly (102 female, 101 male; mean age $=33.1$ years, $S D=14.44)$. Participants were recruited via several means, including through the Department's announcement board, handouts in psychology classes, Facebook university groups, and direct contact with the experimenters. Participants who were enrolled in the university's psychology bachelor's degree classes were 
able to acquire credits as part of their program. For this study, we explicitly recruited 'smoking participants'.

Participants were asked to provide information about their smoking behavior. Only those participants who reported that they were a 'regular cigarette smoker' (at least 1 cigarette per day) were considered for inclusion in this study, mirroring those inclusion criteria applied for studies 1 and 2. Of the 33 excluded subjects, 3 claimed to be strict non-smokers, 23 stated to be casual smokers, and 7 described themselves to be smokers of other tobacco products (e.g. pipe). Additional questionnaires were the same as those used in studies 1 and 2, namely the German version of the Fagerström Test for Nicotine Dependencies (FTND-G) (Schumann et al. 2003), and a questionnaire assessing the subject's attitude toward smoking. Here, participants were asked to respond to ten statements about smoking, indicating their level of agreement toward each one positively (e.g., "smoking is fun”) or negatively (e.g., "smokers smell bad). The questionnaires were not part of our hypotheses and therefore were not considered further.

\section{Materials}

Software and computers. The study was conducted using three mobile testing stations comprising a notebook, a tRNG, and the experimental software. All notebooks used for this study were prepared in an identical fashion. The experimental software was programmed in C\#, and translated the tRNG output into a display of either a smoking-related ('cigarette') picture, or a neutral picture (e.g. objects of daily use). The stimuli were presented in the center of the screen on a black background with a size of 500 x 400 pixels, corresponding to roughly one quarter of the overall screen size. 
Stimuli. All smoking-related pictures were taken out of the Geneva Smoking Photographs (GSP) set (Khazaal, Zullino, \& Billieux 2012), a normative database providing 60 addiction-relevant photographs for nicotine and tobacco research. A set of 10 pictures was chosen from this database, providing variation in terms of product, smoking behavior, and tobacco-related cues (e.g., cigarette packs, ashtrays, smoking individuals, etc.).

All neutral pictures were taken from the International Affective Picture System (IAPS) (Lang, Bradley, \& Cuthbert 1997), a dataset providing an experimental set of 1,169 digitized photographs rated on arousal and valence on a 9-point scale. A set of ten pictures displaying everyday objects and geometric forms was chosen from this dataset. These pictures were rated neutral (mean valence $=4.90, S D=1.09)$, and unexciting (mean arousal $=2.61 ; S D=1.86)$.

Generation of quantum randomness. Randomness in the form of superposed quantum states was provided by a quantum random number generator (Quantis-v10.10.08), as developed by the company ID Quantique (http://www.idquantique.com/randomnumbergeneration/quantis-random-number-generator). This tRNG generates quantum states by emitting photons aimed at a semi-conductive mirror-like prism, similar to the well-known double-slit experiment. Photons have an equal chance of being deflected or passing through the prism to be registered by one of two sensors. Upon measurement, the photon's superposition vanishes, and it is found on either route with a $50 \%$ probability. Depending on which sensor registers the photon, a "0" or " 1 "-bit is created and passed to the computer via a USB interface. The Quantis tRNG has passed all serious tests of randomness (e.g. NIST SP800-22 Compliance) and is considered one of the most effective sources of randomness (Turiel 2007).

\section{Experimenters}

Informally trained research assistants were used as the experimenters for this study; their task was to find 203 participants who were regular smokers. The experimenters only had 
rudimentary knowledge about the aim of the experiment and were blind toward the specific hypothesis at the point of data collection.

\section{Procedure}

Participants were tested in different environments without distractions or other persons present. At the beginning of the experiment written instructions were read to the participants:

Thank you for participating in this experiment! In the first part of the study you will sit in front of the computer and look at pictures. I know that this can be very tiring, nevertheless I ask you not to get distracted and that you focus your attention on the computer for the whole period of this part of the study. It is absolutely necessary for this experiment that you look at the pictures! This will take approximately 10 minutes. Of course, you can quit the experiment at any time, should you feel uncomfortable. As soon as you have finished a message will appear on the screen. Please let me know, so I can prepare the computer for the second part of the experiment, which will be a questionnaire. Filling it out will take about five more minutes. All data will be collected anonymously.

Do you have any questions?

After participants' potential questions were answered, the experimenter opened the software and instructed participant to start with the presentation of the pictures when they felt ready. Subsequently, the experimenters sat aside and distracted themselves mentally to exclude possible interference with the experiment itself, only occasionally checking on the participants.

Participants attentively watched a series of 400 picture trials displaying either an addiction-related or a neutral stimulus on each trial, depending on the output of the tRNG. Stimuli were chosen by sampling without replacement during a ten-trial block. After every 
$10^{\text {th }}$ trial, all pictures had the same probability of being chosen again. This process ensured that, over the course of the experiment, each stimulus had the same probability of being displayed and affecting a participant. Participants first looked at a centered cue (700 msec), then at the addiction-related or neutral stimuli (400 msec), and finally at a black screen (400 msec). This process was repeated 400 times and took approximately 10 minutes (see Figure $3)$.

After the completion of the stimulus presentation, participants were asked to fill out the questionnaire using the computer's web browser.

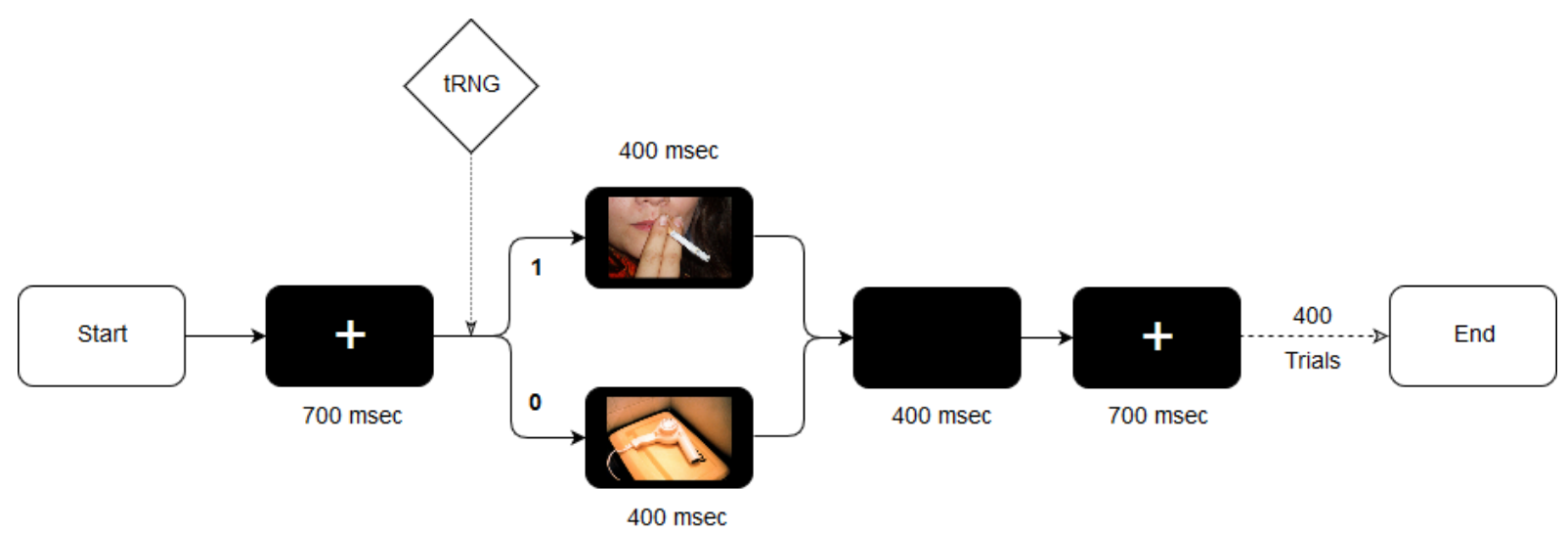

\section{Derivation of the prediction}

Data from studies 1 and 2 were transformed to cumulative z-scores in order to derive a prediction. First, standard deviation was calculated for each of the 298 data points:

$$
S D_{n}=\sqrt{t \times n \times p \times(1-p)}
$$

Where, $\mathrm{t}$ is the number of trials $(\mathrm{t}=400), \mathrm{n}$ is the number of subjects from 1 to 298 , and $p$ is the probability of a hit for a single trial $(p=0.5)$. Subsequently, the difference between the cumulated number of hits (number of smoking-related pictures) from the expected value for each data point was transformed in units of standard deviations:

$$
z_{n}=\frac{\sum_{1}^{n} M_{o b s}-\sum_{1}^{n} M_{e x p}}{S D_{n}}
$$


The resulting data set was then plotted and a nonlinear model fit applied using the function of a general dampened harmonic oscillation:

$$
y(n)=a e^{-\beta n} \cos (\omega n+\varphi)+m n+h
$$

The software used for the curve fit (Wolfram Mathematica 11.1) required boundary values for a model fit this complex; hence, the following boundaries were given:

$$
\begin{aligned}
-5 & <a<5 \\
0 & <b<0.01 \\
0 & <\omega<0.1 \\
0 & <\varphi<2 \pi \\
-5 & <m<5 \\
-5 & <h<5
\end{aligned}
$$

After it had been ensured that none of the fitted parameters were near a constraint boundary, the goodness of the fit was evaluated by a variance estimation using the mean squared error. It was ensured that no other relevant combination of constraint boundaries would produce a lower variance estimation than the attained variance of $\operatorname{Var}=0.065$, and, therefore, a better fit to the experimental data. The final model produced the following estimates:

$\begin{array}{cccc} & \text { Estimate } & \text { Standard Error } & \text { Confidence Interval } \\ a & 1.53484 & 0.0472682 & \{1.44197,1.62771\} \\ \beta & 0.0031827 & 0.000182361 & \{0.0028244,0.00354099\} \\ \omega & 0.0191355 & 0.000296296 & \{0.0185534,0.0197177\} \\ \varphi & 6.00159 & 0.0599918 & \{5.88372,6.11946\} \\ m & 0.00193325 & 0.00014269 & \{0.0016529,0.00221361\} \\ h & -3.02286 & 0.0423804 & \{-3.10613,-2.93959\}\end{array}$

Therefore, we used a damped harmonic oscillation of the following kind to produce the prediction for this study, from $N=298$ to $N=500$ (see also Figure 2).

$$
y(n)=1.53484 e^{-0.0031827 n} \cos (0.0191355 n+6.00159)+0.00193325 n-3.02286
$$

This predictive function was described in the pre-registration stage, as well as in the original Maier and Dechamps (2018) article (p. 288). 


\section{Data analysis}

To determine whether the experimental data of this study fits the prediction, we decided to look at three key characteristics. Firstly, if the courses of the empirical and the prediction curves are similar, the area between them should be minimal. We therefore decided to calculate the area under the curve (AUC) of both the experimental data and the prediction before using their difference as the first test statistic.

Secondly, harmonic oscillations are generally characterized by a systematic upward and downward movement with local minimum and maximum points. Our prediction shows such a local maximum (a maximum for negative effect, so technically a minimum) at $N=429$ and predicts a $\mathrm{z}$-score of $z=-2.70$. We then used the Euclidean distance of the local maximums of the experimental data and the prediction as second test statistic.

Thirdly, we decided to look at the last cumulated data point, since it contains the most precise information about the effect. To test how well our harmonic oscillation predicts the final z-score, we decided to use the Euclidean distance of the end points of the experimental data and the prediction as a third test statistic.

Since no theoretically derived criteria were available that indicated at which respective score these statistics could be defined as being statistically significant, we decided to compare our results with those obtained from 10,000 simulations. Simulation data were generated using the same tRNG device employed in the original study. For each simulation, $203 \mathrm{x} 400$ bits were generated and summed up in the same fashion as in the experimental data set; this produced 10,000 instances of a purely random development of our data which we could compare our actual experimental data. 


\section{Main Results}

Of the 236 subjects, 203 self-identified as regular smokers and were added to the overall analysis, which also included smokers' data from studies 1 and 2 . The total mean for cigarette pictures was $M=199.1(S D=10.11)$. This is slightly below chance value and, therefore, in the same hypothesized direction as in the first two studies. A Bayesian analysis revealed a $B F_{10}=0.40$, which corresponds to anecdotal evidence toward the null hypothesis. Accordingly, in looking at this average score no substantial deviation from chance was found. It must be remembered that we did not hypothesize a consistent effect for the data in this study but rather a specific development of the effect curve.

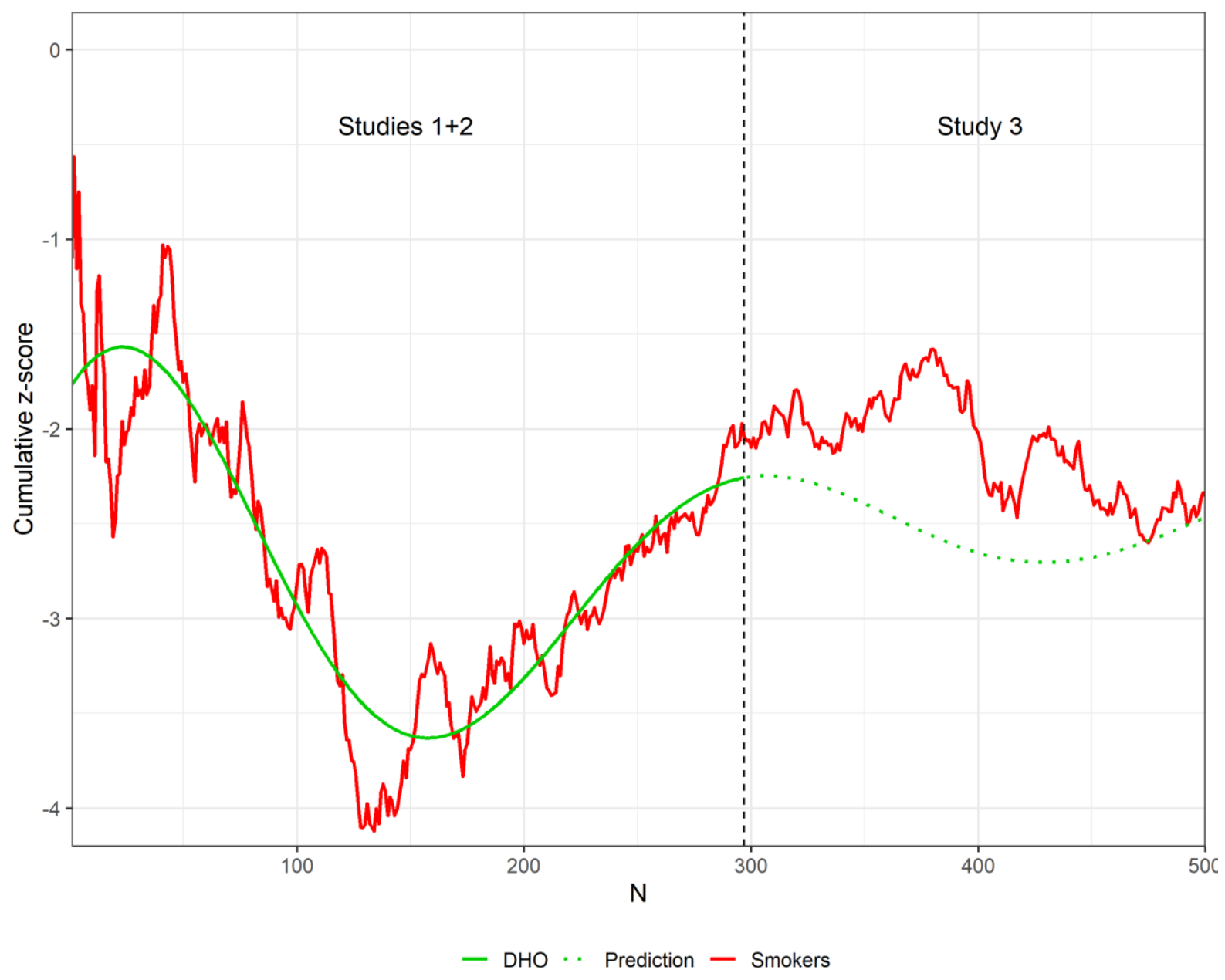

The z-score plot of the experimental data started slightly above the prediction value, before rising toward zero until it reached a z-score of -1.58 at $N=380$. Subsequently, the 
effect can be seen to increase once more, reaching a local maximum at $N=475$ with a z-score of -2.60 . Finally, the last data point at $N=500$ equals $z=-2.35$, which is slightly more negative than at the beginning of Study $3(z=-2.06$; see Figure 4)

Area under curve. The area between the prediction and the experimental data curve was found to be 85.22 . Of all 10,000 simulations, $18.71 \%$ revealed a smaller area between them and the prediction (Mean $A U C=157.48, S D=74.03$ ). Figure 5 shows the distribution of AUCs.

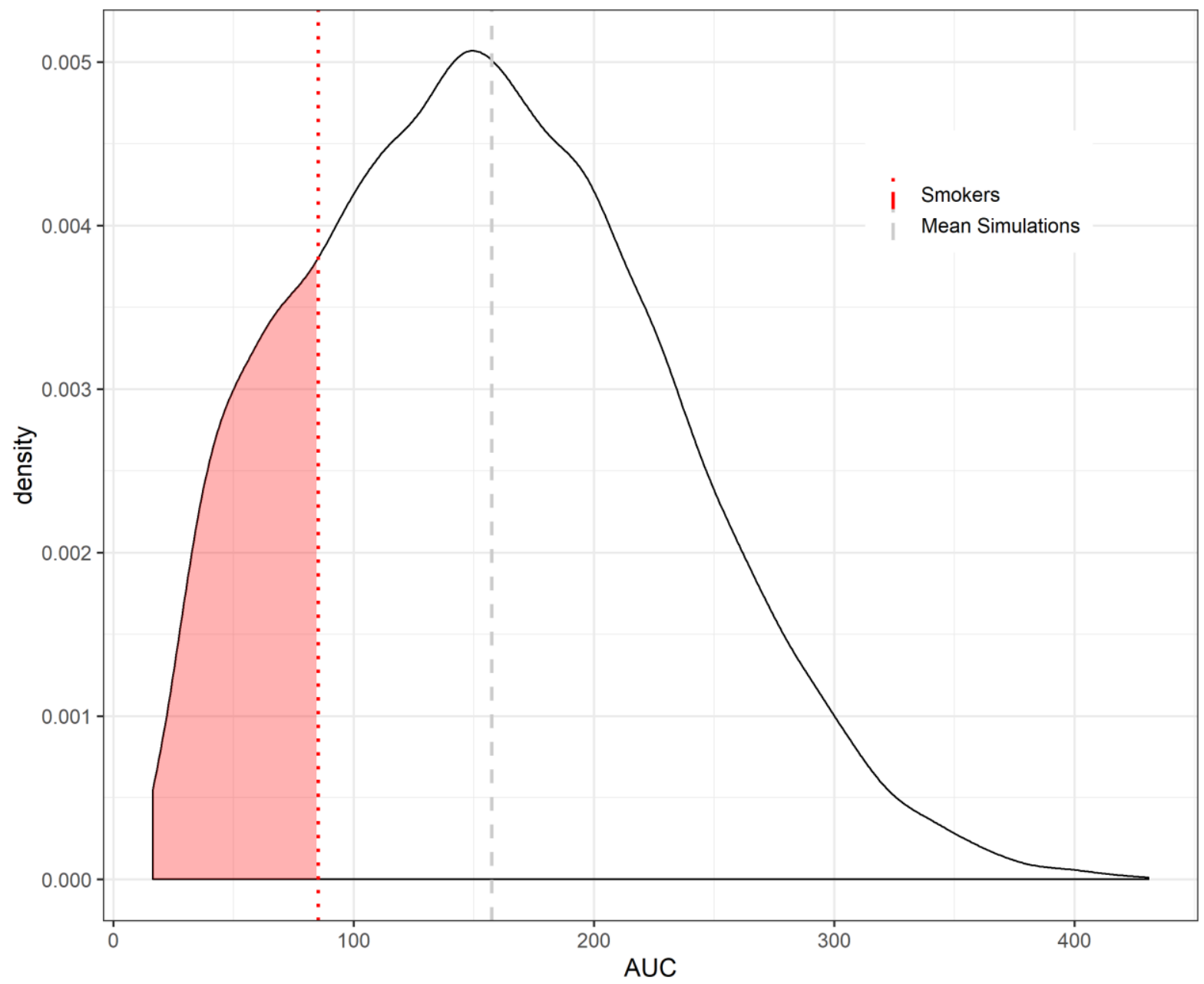

Local maximum (turning point). The local maximum, or turning point, of the experimental data is equivalent to the highest absolute z-score; this was identified at $N=475$ whereby $z_{\max }=-2.60$. To measure the distance to the predicted turning points, $\mathrm{x}-$ and $\mathrm{y}-$ coordinates were standardized to fit in a 1 x 1 square, i.e. the lowest value was transformed so 
that it was equal to 0 , and the highest so that it was equal to 1 . This ensured, that both distances — value of the z-score and the subject number-were accounted for in equal parts. The transformed distance between the turning points of the experimental data and the prediction was $d_{\text {trans }}=0.23$.

In sum, $19.42 \%$ of simulations showed a smaller distance for their turning points to the prediction $($ mean distance trans $=0.44, S D=0.19)($ see Figure 6$)$

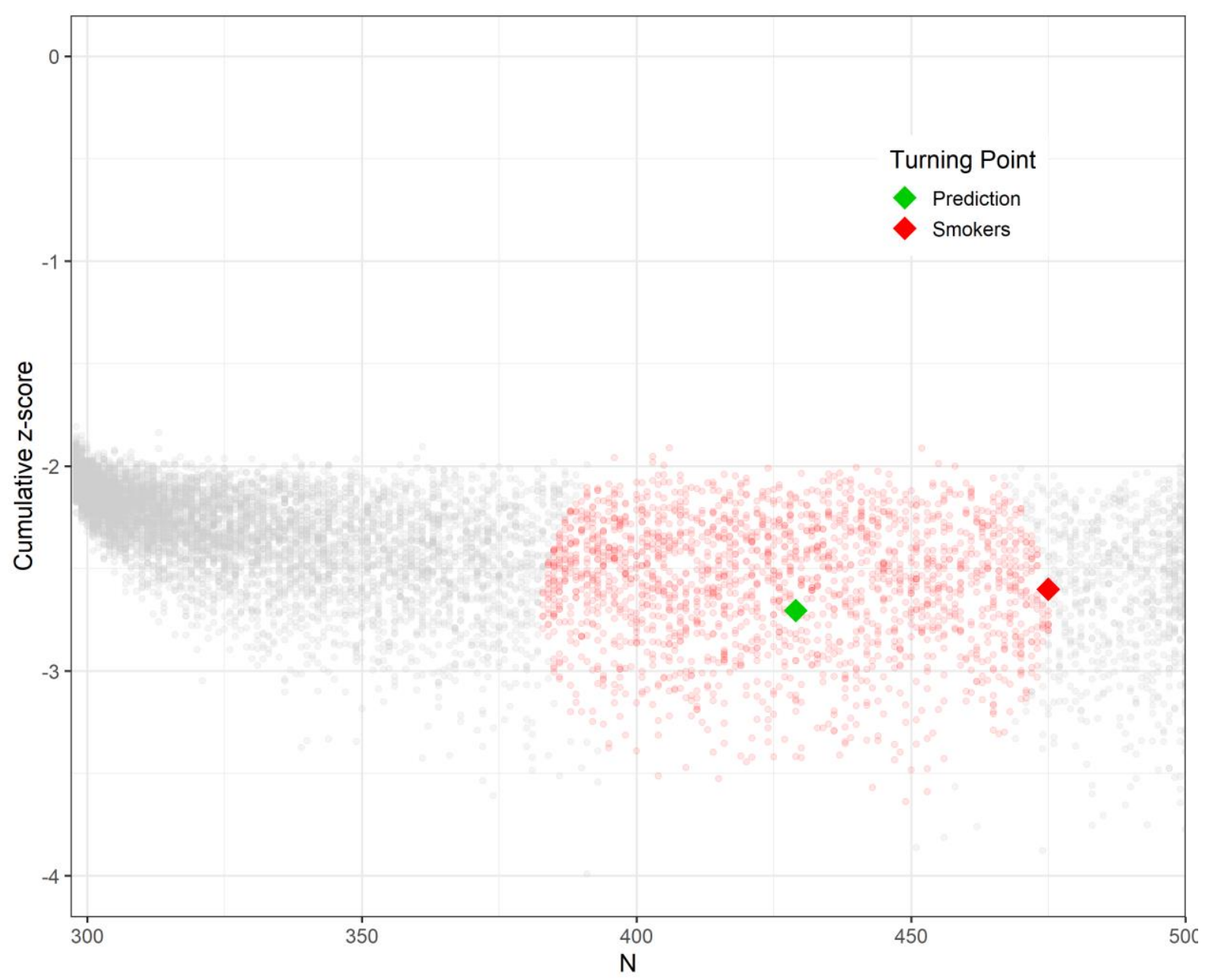

End point. The final z-score of the experimental data lies at $z=-2.35$; this is 0.11 points above the prediction. Of all simulations, only $5.28 \%$ showed a smaller distance to the prediction (mean distance $=0.90, S D=0.62)$. 


\section{Discussion of the main analyses}

The results of our confirmatory analyses of study 3 - which combined data from Maier and Dechamps (2018) with the newly collected data described herein-revealed a mixed picture. For two of the three analyses applied, roughly $1 / 5^{\text {th }}$ of all simulations showed a closer match to our prediction function than the actual experimental data. This includes the area between the curves, as well as the occurrence of the local maximum or turning point.

Descriptively, it seems that the general trend - a temporary increase of the effect that declines towards the end of the study — is present in the data. As can be seen in the median simulation in Figure 7, a truly random dataset should move towards $z=0$. In contrast, while the experimental data do indeed temporarily increase in effect, the highest effect is found 46 subjects later than predicted. The final cumulative z-score, the end point of the curve, is met pretty well by the experimental data. Only $5.28 \%$ of all simulations lie closer to the prediction, which correctly predicted a more extreme value than the initial z-score.

Even though the experimental data are closer to the prediction than most simulations, they do not stand out in a statistically significant way. A closer look at the characteristic of the prediction increases the plausibility of this finding. Although the prediction shows a subtle oscillating pattern, the development is not notably protuberant, meaning that it may not be sufficiently distinctive to be able to reliably distinguish between random and non-random data. Therefore, it remains inconclusive as to whether the temporal development of a microPK effect follows the pattern of a dampened harmonic oscillation closely enough to derive a prediction about its future progress. 


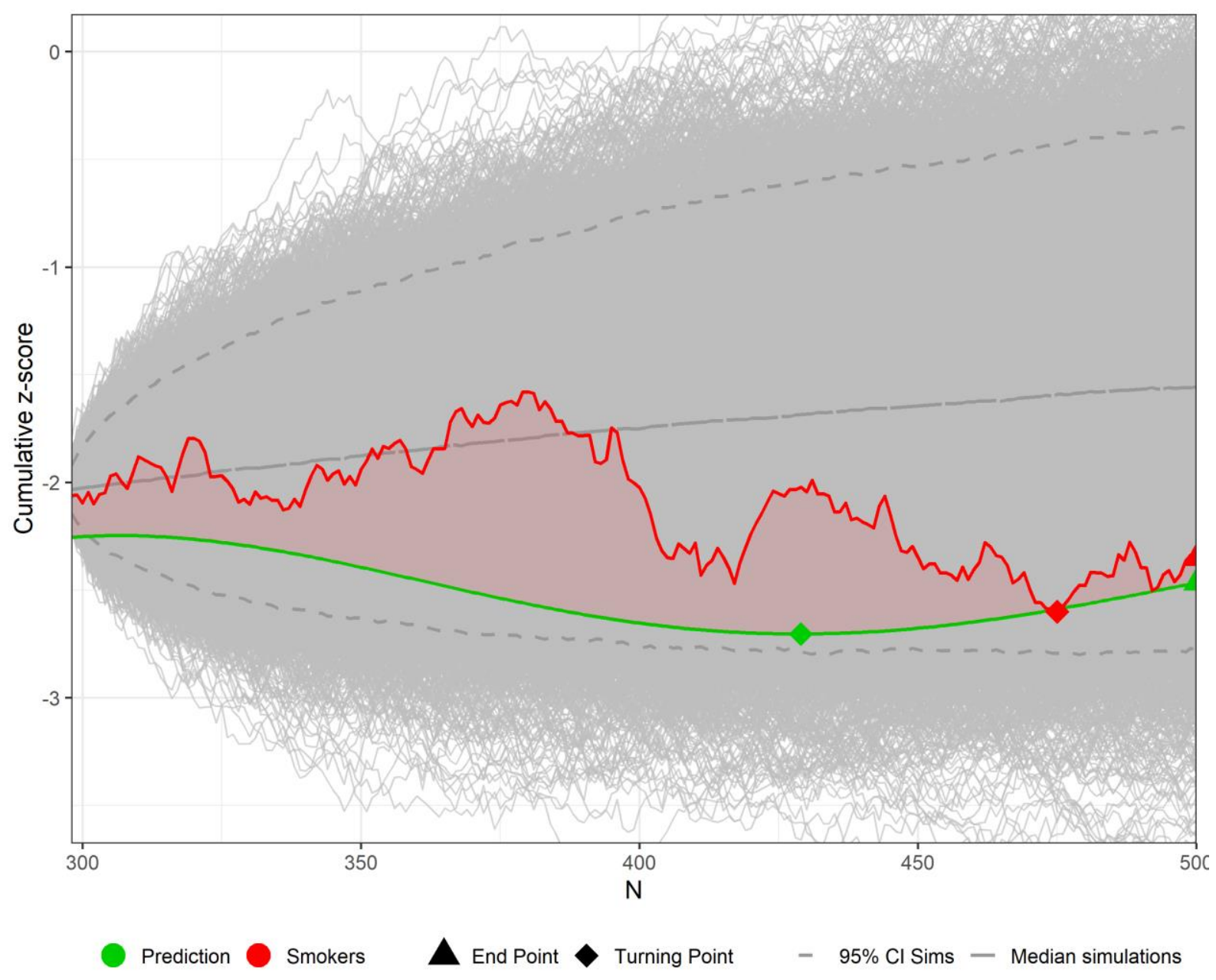

\section{Exploratory Results}

Since the pre-registered confirmatory analyses of study set 3 yielded no definite results, we decided to further explore the unambiguously extraordinary effect development of the smoker subsample over the course of all 500 participants. Arguably, the best indicator of evidence of an effect at any moment is the $B F$. This test statistic can be used sequentially and gives a precise estimate of the probabilities of two competing hypotheses at every data point. The $B F$ is consistent, meaning that it will give a more precise answer the more data it considers, even if the null hypothesis is true (Rouder et al. 2009).

For this reason, we decided to take a closer look at the temporal change of the $B F \mathrm{~s}$ of both subsamples (smokers and non-smokers) before comparing them to another set of simulations. These simulations were generated in the same way as before, though on this 
occasion contained 200,000 bits for a total of 500 data points (400 trials x 500 subjects per simulation). Again, we generated 10,000 simulations using the tRNG device, summed up the bits, and calculated two-sided sequential Bayesian t-Tests with a prior of $\delta \sim$ Cauchy $(0,0.5)$ (see Figure 8)

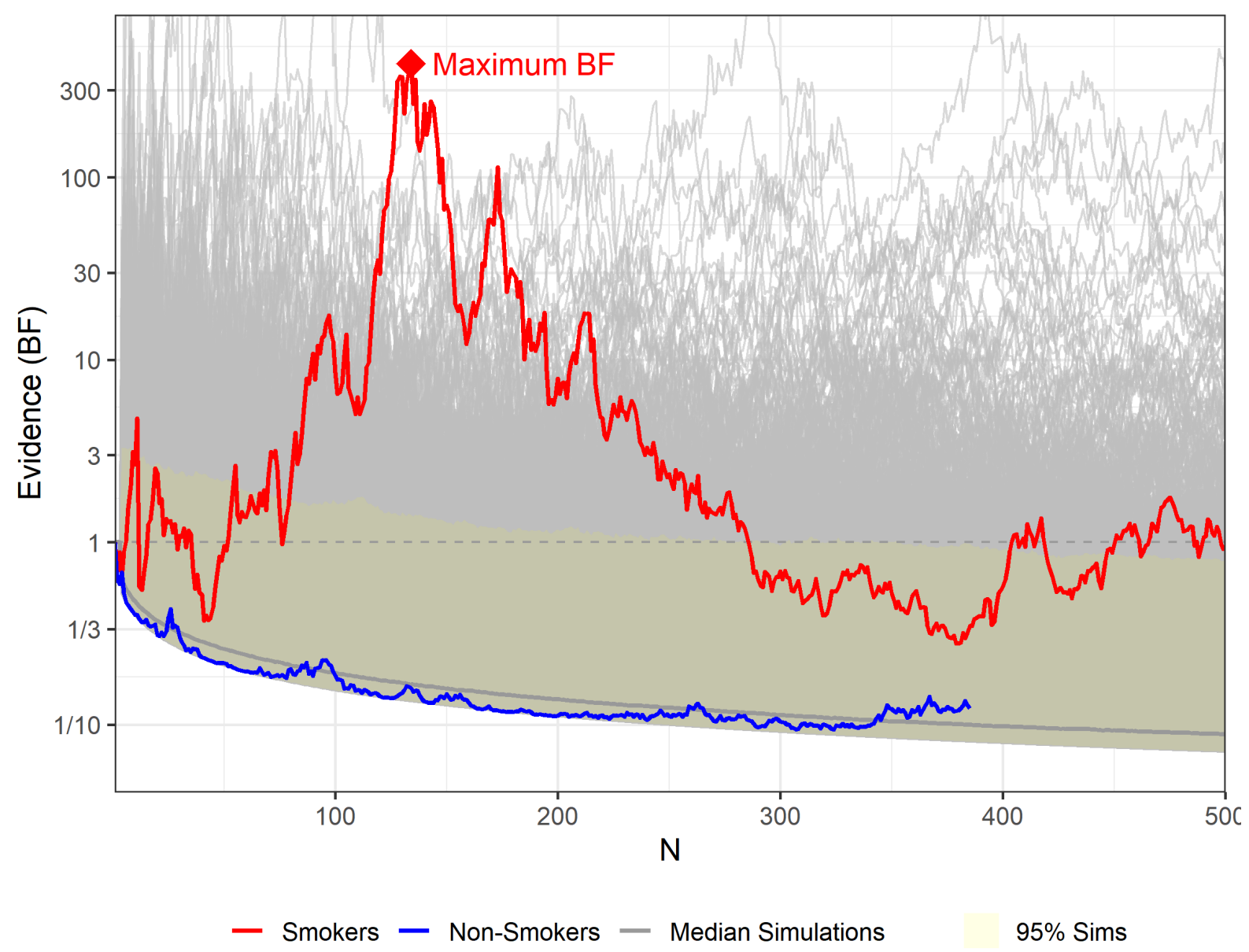

Highest $B F$ reached. As can be seen in Figure 8, the smoker subsample $\left(B F_{10}=421.22\right.$ at $N=134$ ) peak is remarkable. Only $0.21 \%$ of all simulations showed a higher $B F$ at any point. This is in stark contrast to the non-smoker subsample, which resembles a nearly perfect null curve and at no point rises above a value of 1 in the $\mathrm{H}_{1}$ direction (as do $40.56 \%$ of all simulations).

$B F$ Energy. The overall orientation of the curve is also of interest, much like the highest peak. The overall area between the baseline at $B F=1$, and the empirical sequential $B F$-curve above and below that line, where deviations above $B F=1$ receive a positive and 
below a negative sign can be described as its energy (see Figure 9). For the smoker subsample this area was: $A U C_{\text {Smokers }}=7981.73$, which is larger than of $99.85 \%$ of all simulations (mean AUC $=-291.96, S D=1485.19)$. Non-smokers show an Energy of $A U C_{\text {Nonsmokers }}=-323.35$, which is larger than just $4.05 \%$ of simulations cut after the non-smoker $\mathrm{N}$ of 385 (mean AUC for these simulations is $-208.28, S D=1375.05)$.

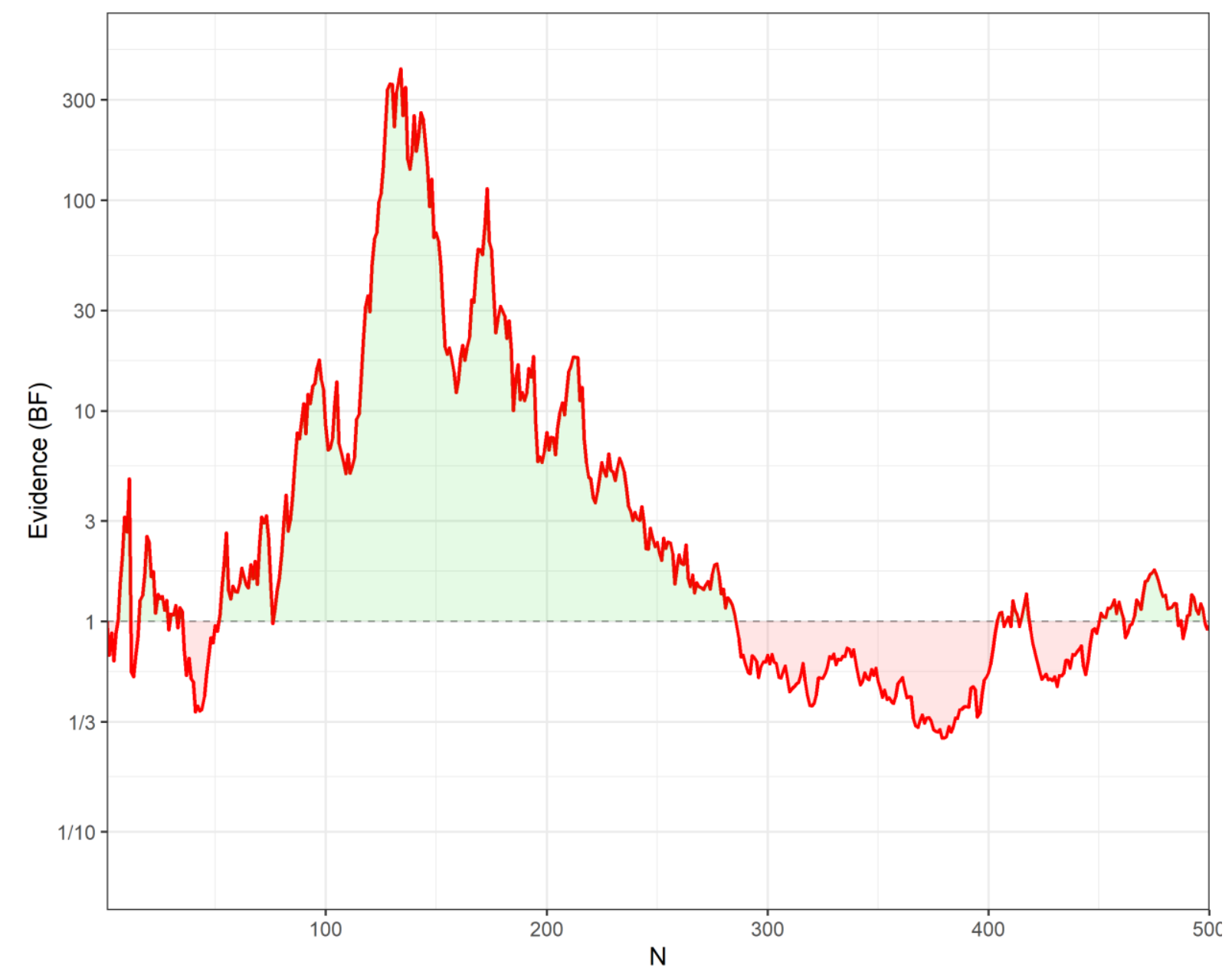

Frequency Spectrum analysis. The Energy of the curve does not account for periodicity as a characteristic however. As this is the core idea of Maier, Dechamps, and Pflitsch (2018) revision of the MPI, which postulates systematic variations of micro-PK effects over time, we then applied an analysis of periodicity to our data set. One such way to gain a deeper understanding of the oscillative nature of our empirical $B F$-curve is by using a Fourier transformation. This analysis converts a time-dependent function to a representation 
of its composited frequencies and can be understood as a harmonic analysis of the input. The resulting curve — the Fourier transform — shows the amplitude of all frequencies comprising the original input sequence (Penrose 2017). Accordingly, high amplitudes indicate the presence of pronounced periodic elements within the original curve.

A fast Fourier transformation (FFT) was conducted on the sequential Bayesian analysis of both subsamples, as well as on all 10,000 simulations. Simulations comparing the non-smoker subsample to were cut after $N=385$ data points. Sampling rate was $1 / \mathrm{N}$ in each case. The transform shows the amplitude at every point of the sampling rate. As it is symmetric, only the first half of the transform is considered. Remarkably, the spectrum analyses of the subsamples differ tremendously: the smoker subsample shows a very high amplitude at almost every frequency (see Figure 10a for the first 50 frequencies); comparatively, the non-smoker subsample shows very small amplitudes (Figure 10b). Compared to the simulation data, 245 of 250 frequencies (98\%) of the transform of the smokers' data have a higher amplitude than $99 \%$ of all simulations, while all frequencies (100\%) have higher amplitudes than $95 \%$ of simulations. Comparatively, none of the frequencies of the non-smoker subsample show an amplitude in the top 5\% of simulations. 

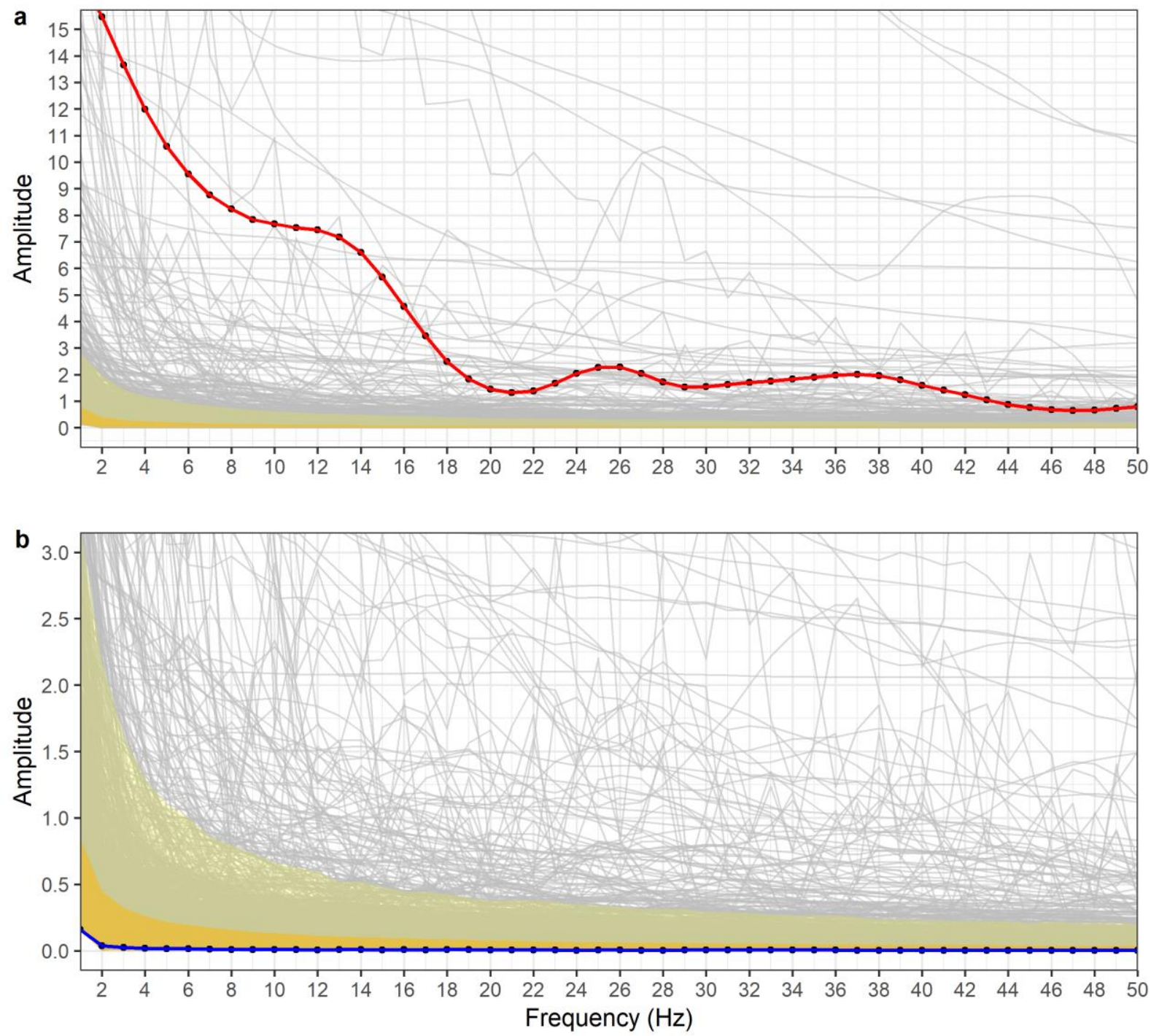

$95 \% \mathrm{Cl}$ sims $\quad 99 \% \mathrm{Cl}$ sims

\section{Discussion of exploratory results}

All exploratory analyses revealed very promising results in their ability to distinguish between the smoker and non-smoker subsamples and simulations. The latter illustrated that the occurrence of a $B F_{10}$ of 421.22 over the course of the data collection stage is quite exceptional; indeed, it can only be expected with a probability of around $0.21 \%$ using truly random data. The $B F$ is designed so that it yields more precise information as more data are added. This strategy reaches a limit though, when the tested effect is not consistent in its strength but volatile, as might be the case with psi effects. 
Considering this, it might be fruitful not only to consider a single $B F$, but also closely examine its development. The difference of the sums of all $B F \mathrm{~s}$ going in the direction of either hypothesis may also provide valuable information; this is calculated by subtracting the correspondent areas from one another, and can be described as the curve's energy. The energy of the sequential Bayesian analysis of the smokers' data is even more unusual than its highest $B F$ and is surpassed by only $0.15 \%$ of all simulations. Conversely, non-smokers' energy resides in the bottom $5 \%$ of simulations. This $B F$ curve is, therefore, oriented remarkably strongly toward the direction of the null hypothesis. The data seems to be 'more random' than most random data sets.

Finally, from our perspective, it is important to account for the possibility of an oscillating nature regarding psi effects. Effects like micro-PK might be possible in general, however, they are likely being counteracted by an opposing force at some point. This interplay should present itself as a periodic pattern of the effect (Maier, Dechamps and Pflitsch 2018). As a Fourier transformation is able to extract the harmonic structure of an input signal, it might therefore be suitable as an instrument to reveal such a non-random oscillating patterns. A remarkable difference can be noticed when this is applied to the sequential Bayesian analyses of both subsamples and their corresponding simulations; the amplitude of the frequencies - meaning their significance in comprising the input signal— show a much higher value in the smoker subsample. When compared to 10,000 simulations, this transform features amplitudes in the top $1 \%$ in nearly every frequency. This means that it is highly unlikely to be obtained from a truly random $B F$ development. Comparatively, the non-smoker transform on the other hand shows a pattern similar to a typical null-effect simulation, and does not stand out in any way.

\section{General Discussion}


A certain degree of experimental evidence for psi effects is unquestionable (Cardeña, 2018). Nevertheless, successful replications of psi effects, like micro-PK effects, are scarce, which is demonstrated exemplarily in the first two studies of this experiment (Maier \& Dechamps 2018). It seems that these effects evade a classical detection through replication. Different ideas toward the elusive nature of psi have emerged, one example of which is the No-Transmission-Axiom of the Model of Pragmatic Information (von Lucadou, Römer, \& Walach 2007). The authors of this model conclude that psi effects in general cannot be tested with specific predictable outputs and are, therefore, not accessible to reproducibility oriented research (von Lucadou, 2006).

A more tangible equivalent to these considerations is grounded on the quantum nature with which psi effects are usually brought into connection. A systematic deviation from quantum randomness violates the No-communication theorem and the Second Law of Thermodynamics because information is created from nothing and could be used to transmit signals. This temporary decrease in entropy could lead to an onset of a counter-mechanism whose task it is to restore randomness, dwindle the information away, and increase entropy. There is one critical difference to the MPI, however: we predict that the interplay between effect and counter-effect leads to a systematic pattern in the development of the effect itself; in principle, this is something that can be predicted and tested (Maier, Dechamps, \& Pflitsch 2018; Maier \& Dechamps 2018).

Our first instinct was to assess the z-score as this embodies the standardized deviation from a given value: in this case, from chance. We hypothesized that effect and counter-effect should balance in a form comparable to a dampened harmonic oscillation and applied a correspondent curve-fitting algorithm to experimental data showing a rise and decline of micro-PK. In doing this we derived a prediction about the behavior of the cumulative z-score of future data. At this point, no definitive conclusion can be drawn regarding the goodness of 
the prediction. Experimental data of 203 further participants in the micro-PK study fitted the prediction better than most simulations according to three different test statistics; however, they did not stand out in a significant way. This is potentially attributable to the lack of distinctiveness, a characteristic that makes it easy for random data to match the prediction.

Subsequently, we aimed to find other ways of assessing the development of the effect on a post hoc basis. We decided to favor the sequential Bayesian analysis to the cumulative zscore because the former represents more sophisticated information regarding the state of effect at every point of the experiment. We felt that the evidence towards one of two hypotheses better matches the knowledgeable information of the presence of an effect, which should comprise the basis for the interplay with the entropic counter-effect. This development went hand in hand with new methodological possibilities that arose during the course of the experiment, specifically, the extraction of individual $B F \mathrm{~s}$ and the execution of FFTs.

Analyzing the development of the $B F$ has proven to be a very promising approach using the data from this series of experiments. The maximum $B F$ expresses the climax of the initially present micro-PK effect, whereas the curve's energy gives an indication of the overall distribution of the $B F \mathrm{~s}$ in regard to the competing hypotheses. Both these methods could reliably distinguish between an experimental sample and a control group or simulated data at a $1 \%$ level. In particular, we would like to emphasize the method of a Fourier transformation of the sequential Bayesian analysis. This approach assesses the existence of a periodicity in the data, which is the fundamental idea behind an interplay of effect and counter-effect. The transform of the experimental sample showed a behavior that fully supports this claim. The remarkably high amplitudes seen for nearly every frequency suggest the presence of a strong oscillative element only in the data set where micro-PK is expected. The transform stands out from both the control group and random simulations. Meanwhile, all three methods have been 
applied to a different high-power and within-subject micro-PK study; once again, they show very promising results (Dechamps \& Maier 2019).

Psi effects such as micro-PK repeatedly present us with scientific challenges. This circumstance has led some to conclude that the classical epistemological standpoint—which demands the replication of results to establish a proof - is not applicable within such a context. While we agree that current standard methods might not be suitable when securing comprehensive evidence for volatile effects, such as psi, we propose a way out without giving up testability and predictability. The goal, therefore, must be to move away from only considering end results and toward a closer investigation of temporary change and the development of psi effects themselves. 


\section{References Cited}

Atmanspacher, H. (2004). Quantum theory and consciousness: An overview with selected examples. Discrete dynamics in Nature and Society, 2004(1):51-73.

Atmanspacher, H. (2012). Dual-aspect monism à la Pauli and Jung. Journal of Consciousness Studies, 19(9-10):96-120.

Atmanspacher, H., Römer, H., \& Walach, H. (2002). Weak quantum theory: Complementarity and entanglement in physics and beyond. Foundations of physics, 32(3):379-406.

Atmanspacher, H., \& Filk, T. (2012). Contra classical causality violating temporal bell inequalities in mental systems. Journal of Consciousness Studies, 19(5-6):95-116.

Bell, J. S. (1964). On the Einstein Podolsky Rosen Raradox. Physics Physique Fizika, 1(3), 195.

Bem, D. J., Palmer, J., \& Broughton, R. S. (2001). Updating the Ganzfeld database: A victim of its own success?. The Journal of Parapsychology, 65(3):207-219.

Bem, D. J., Utts, J., \& Johnson, W. O. (2011). Must psychologists change the way they analyze their data?. Journal of Personality and Social Psychology, 101(4):716-9.

Bösch, H., Steinkamp, F., \& Boller, E. (2006). Examining psychokinesis: The interaction of human intention with random number generators -- A meta-analysis. Psychological bulletin, 132(4):497-523.

Braud, L.W. \& Braud, W. G. (1979). Psychokinetic effects upon a random event generator under conditions of limited feedback to volunteers and experimenter. Journal of the Society for Psychical Research, 50:21-32.

Cardeña, E. (2018). The experimental evidence for parapsychological phenomena: A review. American Psychologist, 73(5):663-677.

Debes, J., \& Morris, R. L. (1982). Comparison of Striving and Non-striving Instructional Sets in a PK Study. Journal of Parapsychology, 46(4):297-312.

Dechamps, M. C., \& Maier, M. A. (2019). Enhancing Micro-PK through Altering Primes. Manuscript in preparation.

Einstein, A., Podolsky, B., \& Rosen, N. (1935). Can quantum-mechanical description of physical reality be considered complete?. Physical review, 47(10):777.

Etzold, E. (2004). Ist die Existenz von Psi-Anomalien beweisbar. Zeitschrift für Anomalistik, 4:1451.

Filk, T., \& Römer, H. (2011). Generalized quantum theory: Overview and latest developments. Axiomathes, 21(2):211-220.

Greenstein, G., \& Zajonc, A. G. (2006). The quantum challenge: Modern research on the 544 foundations of quantum mechanics (2nd ed.). Boston: Jones and Bartlett. 
Hameroff, S. (2012). How quantum brain biology can rescue conscious free will. Frontiers in Integrative Neuroscience, 6(1):93. https://doi.org/10.3389/fnint.2012.00093

Hameroff, S., \& Penrose, R. (1996). Orchestrated reduction of quantum coherence in brain microtubules: A model for consciousness. Mathematics and Computers in Simulation, 40(34):453-480. https://doi.org/10.1016/0378-4754(96)80476-9

Jahn, R., Dunne, B., Bradish, G., Dobyns, Y., Lettieri, A., Nelson, R., Mischo, J., Boller, E., Bösch, H., Vaitl, D., Houtkooper, J., \& Walter, W. (2000). Mind/machine interaction consortium: PortREG replication experiments. Journal of Scientific Exploration, 14(4):499-555.

Kennedy, J. E. (2003). The capricious, actively evasive, unsustainable nature of psi: A summary and hypotheses. Journal of Parapsychology, 67(1):53-74.

Khazaal, Y., Zullino, D., \& Billieux, J. (2012). The Geneva Smoking Pictures: Development and preliminary validation. European Addiction Research, 18(3):103-109.

Lang, P. J., Bradley, M. M., \& Cuthbert, B. N. (1997). International affective picture system (IAPS): Technical Manual and Affective Ratings. NIMH Center for the Study of Emotion and Attention:39-58.

von Lucadou, W. (1995). The model of pragmatic information (MPI). European Journal of Parapsychology, 11:58-75.

von Lucadou, W. (2006, October). Self-Organization of temporal structures - a possible solution for the intervention problem. In AIP conference proceedings, 863(1):293-315.

von Lucadou, W. (2015, July). The correlation-matrix method (CMM) -- a new light upon the repeatability problem of parapsychology. In Paper for the $58^{\text {th }}$ Annual Convention of the Parapsychological Association and $39^{\text {th }}$ SPR International Annual Conference, University of Greenwich.

von Lucadou, W., Römer, H., \& Walach, H. (2007). Synchronistic phenomena as entanglement correlations in generalized quantum theory. Journal of Consciousness Studies, 14(4):50-74.

Maier, M. A., \& Dechamps, M. C. (2018). Observer effects on quantum randomness: Testing Micro-psychokinetic effects of smokers on addiction-related stimuli. J. Scientific Exploration. 32(2):265-297.

Maier, M. A., Dechamps, M. C., \& Pflitsch, M. (2018). Intentional Observer Effects on Quantum Randomness: A Bayesian Analysis Reveals Evidence Against Micro-Psychokinesis. Frontiers in Psychology, 9:379.

Mensky, M. B. (2013). Everett Interpretation and Quantum Concept of Consciousness. NeuroQuantology, 11(1):85-96. 
Neumann, J. V. (1932). Mathematische Grundlagen der Quantenmechanik. Berlin: Julius Springer.

Penrose, R. (1989). The Emperor's New Mind Concerning Computers, Minds and the Laws of Physics. New York: Oxford University Press.

Penrose, R. (1994). Shadows of the Mind: A Search for the Missing Science of Consciousness. Oxford: Oxford University Press.

Penrose, R. (2017). Fashion, Faith, and Fantasy in the New Physics of the Universe. Princeton: Princeton University Press.

Penrose, R., \& Hameroff, S. (2011). Consciousness in the universe: Neuroscience, quantum spacetime geometry and Orch OR theory. Journal of Cosmology, 14:1-17.

Radin, D. (2006). Entangled Minds: Extrasensory Experiences in a Quantum Reality. New York: Simon and Schuster.

Radin, D. I., \& Nelson, R. D. (1989). Evidence for consciousness-related anomalies in random physical systems. Foundations of Physics, 19(12):1499-1514.

Radin, D., Nelson, R., Dobyns, Y., \& Houtkooper, J. (2006). Reexamining psychokinesis: Comment on the Bösch, Steinkamp and Boller (2006) Meta-Analysis. Psychological Bulletin, 132:529-532.

Rouder, J. N., Speckman, P. L., Sun, D., Morey, R. D., \& Iverson, G. (2009). Bayesian t tests for accepting and rejecting the null hypothesis. Psychonomic bulletin \& review, 16(2):225-237.

Schumann, A., Rumpf, H. J., Meyer, C., Hapke, U., \& John, U. (2003). Deutsche Version des Fagerström-Test for Nicotine Dependence (FTND-G) und des Heaviness of Smoking Index (HSI-G). Elektronisches Handbuch zu Erhebungsinstrumenten im Suchtbereich (EHES). Version, 4.

Stapp, H. P. (2015). A quantum-mechanical theory of the mind-brain connection, Beyond Physicalism, ed. by E.F. Kelly et al., Lanham: Rowman and Littlefield:157-193.

Stapp, H. P. (2017). Quantum Theory and Free Will. New York: Springer.

Turiel, T. P. (2007). Quantum random bit generators. The American Statistician, 61(3):255-259.

Varvoglis, M., \& Bancel, P. A. (2015). Micro-Psychokinesis. In E. Cardeña, J. Palmer, \& D. Marcusson-Clavertz (Eds.), Parapsychology: A Handbook for the 21 ${ }^{\text {st }}$ Century: 266-281. Jefferson NC: McFarland \& Company, Inc., Publishers. 
Walach, H., von Lucadou, W., \& Römer, H. (2014). Parapsychological phenomena as examples of generalized nonlocal correlations-A theoretical framework. Journal Scientific Exploration, 28(4), 605-631.

Wagenmakers, E.-J., Wetzels, R., Borsboom, D., and van der Maas, H. L. J. (2011). Why psychologists must change the way they analyze their data: the case of psi: comment on Bem (2011). Journal of Perssonality and Social Psychology. 100:426-432.

Weizsäcker, E. V. (1974). Erstmaligkeit und Bestätigung als Komponenten der pragmatischen Information. E. v. Weizsäcker (Hrg.): Offene Systeme I:82-113.

Wigner, E. P. (1963). The problem of measurement. American Journal of Physics, 31(1):6-15 


\section{Figure Captions}

Figure 1. Sequential Bayesian analyses of mean number of cigarette pictures for smoker and nonsmoker subsamples. Dotted lines indicate the transition from Study 1 to Study 2 within both subsamples.

Figure 2. Cumulative z-score of the smoker subsample, fitted dampened harmonic oscillation (DHO), and prediction for the following 203 participants.

Figure 3. Experimental procedure: Schematic flow chart for a single trial.

Figure 4. Results of study 3 were transformed into cumulative z-scores and compared to the prediction, the extrapolation of the fitted dampened harmonic oscillation (DHO).

Figure. 5. Distribution of the area under the curve between the simulations and the prediction. Red area indicates the proportion of simulations with a smaller area, i.e. a better fit than the experimental data.

Figure 6. Overview of the turning points (local maxima) of all simulations. Red dots indicate simulations with a turning point closer to the prediction than the experimental data.

Figure 7. Overview of all confirmatory analyses conducted for study 3 . Here, the grey lines represent random simulations; their median is the center grey dashed line, while the outer grey dashed lines represent the borders in which $95 \%$ of all simulations lie. The red line represents smokers' experimental data, and the green line the prediction. Read area visualizes the area under the curve between the experimental data and the prediction. Both turning and end points of the experimental data and the predictions are represented by the rhomb and triangle symbol.

Figure 8. Sequential Bayesian analyses of both subsamples (studies 1-3) in comparison to 10,000 simulations comprised of random data (grey lines). Overall, $95 \%$ of simulations are located in the yellow area; the median of the simulations is represented by the single bottom grey line.

Figure 9. Representation of the curve's Energy. Parts below BF=1 (red; direction towards $\mathrm{H}_{0}$ ) are subtracted from parts above 1 (green; direction towards $\mathrm{H}_{1}$ )

Figure 10. FFT of Bayesian sequential analyses of the smoker subsample (red) and 10,000 simulations, and the non-smoker subsample (blue) and 10,000 simulations. The orange (95\%) and yellow (99\%) areas represent the simulations' empirical confidence bands. 
Table 1. Mean number of cigarette pictures and $B F$ for both subsamples in studies 1 and 2.

\begin{tabular}{rcccccc}
\hline \multicolumn{3}{c}{ Smokers } & \multicolumn{3}{c}{ Non-Smokers } \\
\hline Study 1 & 122 & $196.7(9.87)$ & 66.06 & 132 & $200.5(9.86)$ & 0.16 \\
Study 2 & 175 & $200.3(10.38)$ & 0.09 & 220 & $199.0(10.34)$ & 0.30 \\
Studies 1-2 & 297 & $198.8(10.31)$ & 0.61 & 352 & $199.6(10.11)$ & 0.12 \\
\hline
\end{tabular}


Table 2. Results of confirmatory analyses of study 3 in comparison to 10,000 random simulations.

\begin{tabular}{rcc}
\hline & Result & $\begin{array}{c}\text { \% of simulations with a better } \\
\text { result }\end{array}$ \\
\hline Area under curve & 85.22 & 18.71 \\
Turning Point & 0.23 & 19.42 \\
End Point & 0.11 & 5.28 \\
\hline
\end{tabular}

